\title{
Cesarean Section
}

National Cancer Institute

\section{Source}

National Cancer Institute. Cesarean Section. NCI Thesaurus. Code C46088.

Surgical delivery of one or more intrauterine fetuses though an abdominal incision. 